Revue d'histoire du XIXe siècle

Société d'histoire de la révolution de 1848 et des

révolutions du XIXe siècle

La Restauration revisitée - Les formes de la protestation - Une histoire de l'Etat

\title{
Hervé Guillemain, Diriger les consciences. Guérir les âmes. Une histoire comparée des pratiques thérapeutiques et religieuses (1830-1939), Paris, La Découverte, 2006, 347 p. ISBN : 2707148504. 26 euros.
}

Nicole Edelman

\section{OpenEdition}

Journals

Édition électronique

URL : http://journals.openedition.org/rh19/1982

DOI : $10.4000 /$ rh 19.1982

ISSN : $1777-5329$

Éditeur

La Société de 1848

Édition imprimée

Date de publication : 20 décembre 2007

Pagination : $161-208$

ISSN : 1265-1354

Référence électronique

Nicole Edelman, « Hervé Guillemain, Diriger les consciences. Guérir les âmes. Une histoire comparée des pratiques thérapeutiques et religieuses (1830-1939), Paris, La Découverte, 2006, 347 p. ISBN :

2707148504. 26 euros. », Revue d'histoire du XIXe siècle [En ligne], 35 | 2007, mis en ligne le 03 janvier 2008, consulté le 22 septembre 2020. URL : http://journals.openedition.org/rh19/1982 ; DOI : https:// doi.org/10.4000/rh19.1982 
de l'île de la Cité est particulièrement révélateur. Enfin, cette symbolique atteint un apogée avec les «cathédrales martyres» de la guerre 14-18.

Ainsi, à travers des études de cas autour d'un monument, d'un projet, d'un débat ou d'un homme, Jean-Michel Leniaud, prolongeant une réflexion engagée dans des ouvrages antérieurs, fait du lieu cultuel un lieu de mémoire, naturellement ambivalent car au carrefour de diverses influences : entre Église et État, entre religion et nation, entre foi et culture. Si le propos possède une forte cohérence, on regrettera des redites et répétitions assez nombreuses, défaut que l’on doit imputer au genre du recueil d'articles.

Comme il est exposé dans l'introduction, "la révolution des signes», c'est une période : celle qui tend à incarner de manière visible les ambitions pastorales et ses fondements théologiques et ce, en parfait accord avec la sensibilité du temps. Au style épuré et fortement spiritualisé du début du XIX"e, succède vers 1830 un art religieux orienté vers le sensible. Les années 1920 ouvrent une période nouvelle, qui, avec l'abstraction, renoue d'une certaine manière avec ce qui précédait la période romantique. «Révolution» est donc tout autant à comprendre comme un bouleversement que comme un cycle.

$$
\text { Sylvain Milbach }
$$

\section{Hervé GUILLEMAIN, Diriger les consciences. Guérir les âmes. Une histoire comparée des pratiques thérapeutiques et religieuses (1830-1939), Paris, La Découverte, 2006, 347 p. ISBN : 2707148504. 26 euros.}

L'historiographie de la folie, ouverte en 1961 avec l'ouvrage de Michel Foucault, Histoire de la folie à l'âge classique, n'a cessé de prendre de l'ampleur ces dernières années, au cours desquelles de nouveaux et nombreux travaux nous ont été proposés. Le livre d'Hervé Guillemain s'inscrit parmi ces nouvelles approches et souligne une fois encore la complexité des concepts et des pratiques thérapeutiques psychiatriques. Issu d'une thèse, Diriger les consciences. Guérir les âmes s'attache à l'étude de toutes les directions de la conscience - qu'elles qualifient le sens du mal ou qu'il s'agisse des thérapeutiques interrogées à travers le prisme du religieux chrétien, catholique ou protestant. Ce fil conducteur permet à Hervé Guillemain de parcourir la longue chronologie choisie et d'exposer en l'analysant la sécularisation de la représentation du psychisme qui mène de l'âme aux centres cérébraux. "Avant 1860, le religieux paraît omniprésent dans les asiles publics et privés, mais aussi hors l'asile, les croyances étant au cœur des pratiques de l'exorcisme et du magnétisme. Dans la seconde moitié du siècle, c'est l'ensemble des esprits qui est médicalisé. L'argument médical pénètre au cœur de l'Église comme dans le reste de la société. Le médecin prend progressivement la place du prêtre et devient expert de l'âme et directeur de consciences, en même temps qu'il prend place dans les hôpitaux, dans les institutions républicaines et promeut la laïcisation.» Cette histoire n'en est pas pour autant linéaire : bien au contraire, elle se compose de croisements constants entre laïcs et clercs, entre matérialistes et spiritualistes, d'interactions entre médecins chrétiens et médecins athées mais aussi entre médecins chrétiens, prêtres et théologiens qui parfois sont d'ailleurs une seule et même personne. La mise au jour et l'analyse de ces enchevêtrements est l'une des richesses de ce livre. À travers ces regards successifs, 
Hervé Guillemain scrute les asiles laïcs et privés, catholiques et protestants; il étudie aussi bien le quotidien des formes de soins que les conceptions de l'aliénation et les formes de maladies mentales ou encore les grands phénomènes de la possession, des stigmates et de l'obsession, nettement distingués les uns des autres.

L'ouvrage s'organise autour de trois grands moments conceptuels. Le premier mène des années 1830 à celles de 1860 lorsque l'aliénisme, l'asile et son «traitement moral » prennent forme et se confrontent au christianisme qui en retour et à son tour pense la folie au prisme de cette nouvelle approche médicale. Le problème du diable et de la possession est ainsi revisité. Le deuxième temps - 1860-1900 - est ponctué par la question de l'hypnose qui est mise au jour et à la mode dans les années 1880 et celle de l'hystérie qui stigmatise les saintes, les miracles et les visions. Les dernières décennies enfin - 1900-1939 - voient l'émergence des nouvelles disciplines que sont la psychologie et la psychanalyse et marquent une mutation thérapeutique et un renouveau religieux. Dans cette dernière partie, Hervé Guillemain propose en particulier une nouvelle interprétation des résistances françaises à la psychanalyse par l'analyse de quelques figures de psychologues, philosophes, médecins, prêtres et psychanalystes et nous fait ainsi découvrir le rôle largement ignoré jusque-là de certains chrétiens dans la diffusion de la psychanalyse en France. Il souligne la profondeur de l'étude des relations entre christianisme, psychanalyse freudienne et psychologie, dans De l'angoisse à l'extase de Pierre Janet.

Le livre est ainsi riche en personnalités connues ou inconnues et en découvertes d'asiles religieux jusque-là peu connus; ainsi l'étude des réalisations des frères de Saint-Jean de Dieu à travers les deux figures de Paul de Magallon et de l'étonnant frère Hilarion Tissot. Le volet protestant n'est pas oublié : Hervé Guillemain nous donne à voir la fondation de l'asile de La Force par John Bost, héritier du "Réveil». Les thérapeutiques aliénistes sont décrites, anciennes et nouvelles. L'exorcisme est revisité par le savoir psychiatrique au tournant des $\mathrm{XIX}^{\mathrm{e}}$ et $\mathrm{XX}^{\mathrm{e}}$ siècles par les pères Haza et de Tonquédec. Les grands aliénistes catholiques sont eux aussi bien sûr présents : Jean-Pierre Falret et son disciple Benedict A. Morel trouvent leur juste place au coeur de la révolution conceptuelle de la nouvelle discipline à laquelle ils apportent une couleur religieuse. Hervé Guillemain croise en effet en permanence savoir scientifique et concept religieux. Il étudie précisément le rôle de la société médicale Saint Luc, Saint Côme et Saint Damien fondée en 1884 et par là même les liens entre foi et science, entre médecine scientifique et religion. Il souligne ainsi qu'avec le pontificat de Léon XIII et le renouveau thomiste, la science change d'image dans le monde catholique, ce dont la prolifération des écrits sur les relations entre science et foi témoigne. Il analyse la querelle de l'hypnotisme qui modifie l'image de la conscience et montre combien les découvertes médicales en matière de neurologie provoquent l'ouverture d'une brèche dans le corpus doctrinal du christianisme en relativisant la conscience et le libre-arbitre.

Le foisonnement du livre, adossé aux multiples et très riches sources retrouvées par Hervé Guillemain, découvre ainsi tout un pan de l'histoire psychiatrique jusquelà largement ignorée. Diriger les consciences. Guérir les âmes nous montre avec érudition et subtilité la complexité des entremêlements constants entre science, religion et thérapeutique, toujours présents et toujours changeants. 